\title{
CONTINUOUS DEPENDENCE ON DATA FOR QUASIAUTONOMOUS NONLINEAR BOUNDARY VALUE PROBLEMS
}

\author{
N. APREUTESEI
}

Received 20 December 2003

We devote this paper to quasiautonomous second-order differential equations in Hilbert spaces governed by maximal monotone operators. Some bilocal boundary conditions are associated. We discuss the continuous dependence of the solution both on the operator and on the boundary values. One uses the methods of nonlinear analysis. Some applications to internal approximate schemes are given.

\section{Introduction}

The main purpose of this paper is to prove the continuous dependence on $A, a, b, f$ of the solution of the second-order evolution equation

$$
p u^{\prime \prime}(t)+r u^{\prime}(t) \in A u(t)+f, \quad \text { a.e. } t \in(0, T),
$$

subject to the two-point boundary condition

$$
u(0)=a, \quad u(T)=b .
$$

Here $A: D(A) \subseteq H \rightarrow H$ is a maximal monotone operator (possibly multivalued) in a real Hilbert space $H, D(A)$ is its domain, $a, b \in D(A), f \in L^{2}(0, T ; H)$, and $p, r$ are two continuous functions from $[0, T]$ to $\mathbb{R}$.

In $[10,11]$, Barbu proved the existence of the solution in the case $p \equiv 1, r \equiv 0$. The author considered the boundary value problems

$$
\begin{gathered}
u^{\prime \prime}(t) \in A u(t)+f(t), \quad \text { a.e. } t \in(0, T), \\
u(0)=a, \quad u(T)=b, \\
u^{\prime \prime}(t) \in A u(t), \quad \text { a.e. } t \in(0, \infty), \\
u(0)=a, \quad \sup _{t \geq 0}\|u(t)\|<\infty
\end{gathered}
$$

where $a, b \in D(A)$ and $f \in L^{2}(0, T ; H)$. Denoting by $u_{a}$ the solution of $(1.4)$ and by $S_{1 / 2}(t)$ the extension to the closure $\overline{D(A)}$ of $D(A)$ of the mapping $a \mapsto u_{a}(t)$, one obtains a semigroup of nonlinear contractions $\left\{S_{1 / 2}(t), t \geq 0\right\}$ on $\overline{D(A)}$. Let $-A_{1 / 2}^{0}$ be its infinitesimal 
generator and $A_{1 / 2}$ the unique extension of $A_{1 / 2}^{0}$ to a maximal monotone operator. The operator $A_{1 / 2}$ is called the square root of $A$. Regularity properties of $S_{1 / 2}(t)$ are presented in $[10,11,13]$.

Brézis [14] replaced the condition $u(0)=a$ in (1.4) by $u^{\prime}(0) \in \partial j(u(0)-a)$, where $\partial j$ is the subdifferential of a lower-semicontinuous, convex, and proper function $j: H \rightarrow \overline{\mathbb{R}}$. Problems (1.3) and (1.4) were also studied in Banach spaces [21, 22, 23]. The semigroup $\left\{S_{1 / 2}(t), t \geq 0\right\}$ is defined in this case too [22]. Other contributions to the theory of second-order differential equations (1.3) and (1.4) can be found in $[16,18,19,24,25,26]$.

Aftabizadeh and Pavel [1,2] generalized problem (1.3) to (1.1) with the boundary condition

$$
u^{\prime}(0) \in \alpha(u(0)-a), \quad-u^{\prime}(T) \in \beta(u(T)-b)
$$

with $\alpha, \beta$ being maximal monotone operators in $H$. If we take $\alpha=\beta=\partial j$, where $j: H \rightarrow$ $\overline{\mathbb{R}}$,

$$
j(x)= \begin{cases}0, & x=0, \\ +\infty, & \text { otherwise }\end{cases}
$$

then $D(\partial j)=\{0\}$ and thus (1.5) becomes (1.2). A more general boundary condition can be found in [4]. Antiperiodic solutions for a particular case of (1.1) are given in [3]. In [12], another extension of the equation in (1.3) is studied under a boundary condition of subdifferential type.

As a consequence of [1, Theorem 3.2], it follows that if $A$ is maximal monotone in $H$, $a, b \in D(A), f \in L^{2}(0, T ; H)$ and $p, r:[0, T] \rightarrow \overline{\mathbb{R}}$ are continuous functions, $p(t) \geq c>0$ on $[0, T]$, then problem (1.1)-(1.2) has a unique solution $u \in W^{2,2}(0, T ; H)$.

Discrete variants of (1.3) and (1.4) are studied in [20].

In [5], it is shown that the application which associates to $\{A, a, b\}$ the unique solution $u$ of (1.3) with $f \equiv 0$ is continuous in the following sense. Consider the boundary value problem (1.3) (with $f \equiv 0$ ) and the sequence of problems

$$
\begin{array}{cc}
u_{n}^{\prime \prime}(t) \in A^{n} u_{n}(t), & \text { a.e. } t \in(0, T), \\
u_{n}(0)=a_{n}, & u_{n}(T)=b_{n},
\end{array}
$$

where $A, A^{n}$ are maximal monotone operators in $H, a, b \in \overline{D(A)}, a_{n}, b_{n} \in \overline{D\left(A^{n}\right)}$ with $0 \in A 0 \cap A^{n} 0$. If $a_{n} \rightarrow a, b_{n} \rightarrow b$ in $H$ and

$$
\left(I+\lambda A^{n}\right)^{-1} \xi \longrightarrow(I+\lambda A)^{-1} \xi \quad \text { as } n \longrightarrow \infty,
$$

for all $\xi \in H$, and for all $\lambda>0$, then the solution $u_{n}$ of (1.7) converges to the solution $u$ of (1.3) (with $f \equiv 0$ ), uniformly on $[0, T]$.

In [6], we have a similar result on $(0, \infty)$. The case of the first-order differential equations is analyzed in $[7,15]$. The continuous dependence on data for the antiperiodic solutions to a class of second-order evolution equations with constant coefficients is given in [3]. 
If $A^{n}$ and $A$ satisfy condition (1.8), we say that $A^{n}$ converges to $A$ in the sense of the resolvent. This and other types of convergences of the sequences of operators can be found in [8]. They are of physical interest because of their applications in the homogenization theory, singular perturbation problems, convergence problems in optimal control, stochastic optimization, and so forth. In [22], the authors show that in Banach spaces with some specific properties, the convergence in the sense of resolvent of a sequence $\left(A^{n}\right)$ to $A$ implies the convergence of $\left(A_{1 / 2}^{n}\right)$ to $A_{1 / 2}$ in the same sense.

In the present paper, we prove that the unique solution $u$ of problem (1.1)-(1.2) depends strongly continuous on the data $A, a, b, f$. More exactly, we take the sequence of evolution equations

$$
p u_{n}^{\prime \prime}(t)+r u_{n}^{\prime}(t) \in A^{n} u_{n}(t)+f_{n}, \quad \text { a.e. } t \in(0, T),
$$

subject to the boundary conditions

$$
u_{n}(0)=a_{n}, \quad u_{n}(T)=b_{n}
$$

Here $\left(A^{n}\right)$ is a sequence of maximal monotone operators in $H, a_{n}, b_{n} \in D\left(A^{n}\right)=$ the domain of $A^{n}, f_{n} \in L^{2}(0, T ; H)$. We show that, under some additional conditions, if $a_{n} \rightarrow a$, $b_{n} \rightarrow b$ in $H, f_{n} \rightarrow f$ in $L^{2}(0, T ; H)$, and $\left(A^{n}\right)$ converges to $A$ in the sense of the resolvent, then the solution $u_{n}$ of (1.9)-(1.10) converges in $C([0, T] ; H)$ to the solution $u$ of (1.1)-(1.2).

Using an idea from $[1,2]$, in the next sections, one uses the weighted space $\mathscr{L}=$ $L_{\widetilde{r} / p}^{2}(0, T ; H)$, where

$$
\tilde{r}(t)=\exp \left(\int_{0}^{t} \frac{r(s)}{p(s)} d s\right), \quad t \in[0, T] .
$$

Therefore, the scalar product in $\mathscr{L}$ is

$$
\langle u, v\rangle=\int_{0}^{T} \frac{\tilde{r}(t)}{p(t)}(u(t), v(t)) d t \quad \forall u, v \in L^{2}(0, T ; H),
$$

and the corresponding norm is

$$
|u|=\left(\int_{0}^{T} \frac{\tilde{r}(t)}{p(t)}\|u(t)\|^{2} d t\right)^{1 / 2} \quad \forall u \in L^{2}(0, T ; H),
$$

where $(\cdot, \cdot)$ and $\|\cdot\|$ are the scalar product and the norm of $H$, respectively. Actually, the spaces $L^{2}(0, T ; H)$ and $\mathscr{L}$ contain the same functions and have equivalent norms. The difference between them is that the operator

$$
\begin{gathered}
B u=-p u^{\prime \prime}-r u^{\prime}=-\frac{p}{\widetilde{r}}\left(\tilde{r} u^{\prime}\right)^{\prime}, \\
D(B)=\left\{u \in W^{2,2}(0, T ; H), u(0)=a, u(T)=b\right\}
\end{gathered}
$$


is maximal monotone only in $\mathscr{L}$ (see [1]). Taking into account this remark, we may write (1.1) in the form

$$
\frac{p}{\widetilde{r}}\left(\tilde{r} u^{\prime}\right)^{\prime} \in A u+f, \quad \text { a.e. } t \in(0, T) .
$$

In Section 2, we recall some definitions and results from the theory of maximal monotone operators. The main result is stated in Section 3 and proved in Section 4. The proof combines an idea related to the case $p \equiv 1, r \equiv 0, f \equiv 0$ (see [5]) with some techniques from the existence theory (see $[1,2])$. In the last section, we give a numerical approximation of (1.1)-(1.2) with $f \equiv 0$ by an internal approximating scheme (see [9]).

\section{Preliminaries}

Throughout this paper, $H$ is a real Hilbert space of norm $\|\cdot\|$ and scalar product $(\cdot, \cdot)$. Denote by " $\rightarrow$ " and " $\rightarrow$ " the strong and the weak convergence in all the involved spaces, respectively.

The nonlinear multivalued operator $A$ with the domain $D(A)$ and the range $R(A)$ is said to be monotone if $\left(y_{1}-y_{2}, x_{1}-x_{2}\right) \geq 0$ for all $y_{i} \in A x_{i}, x_{i} \in D(A), i=1,2$. The operator $A$ is called maximal monotone if it is monotone and it has not any proper monotone extension. It is known that $A$ is maximal monotone if and only if $A$ is monotone and $R(A+\lambda I)=H$ for all $\lambda>0$ (or equivalently, for some $\lambda>0$ ) (see [13, Theorem 1.2, page 39]). For $x \in D(A)$, let $A^{0} x$ be the element of least norm in $A x$, that is,

$$
\left\|A^{0} x\right\|=\inf \{\|y\|, y \in A x\} .
$$

The single-valued operator $A^{0}$ which associates to each $x \in D(A)$ the element $A^{0} x$ is called the minimal section of $A$. For every maximal monotone operator $A$, one can define the resolvent $J_{\lambda}$ and the Yosida approximation $A_{\lambda}$ of $A$, namely,

$$
J_{\lambda}=(I+\lambda A)^{-1}, \quad A_{\lambda}=\frac{I-J_{\lambda}}{\lambda}, \quad \lambda>0 .
$$

The realization of $A$ in $L^{2}(0, T ; H)$ is the operator $\mathscr{A}$ given by

$$
\begin{gathered}
D(\mathscr{A})=\left\{u \in L^{2}(0, T ; H), u(t) \in D(A) \text { a.e. on }(0, T),\right. \\
\left.\exists v \in L^{2}(0, T ; H) \text { such that } v(t) \in A u(t) \text { a.e. on }(0, T)\right\}, \\
\mathscr{A} u=\left\{v \in L^{2}(0, T ; H), v(t) \in A u(t) \text { a.e. on }(0, T)\right\} .
\end{gathered}
$$

If $A$ is maximal monotone in $H$, then $\mathscr{A}$ is maximal monotone in $L^{2}(0, T ; H)$. If $A_{\lambda}$ and $\mathscr{A}_{\lambda}$ are Yosida approximations of $A$ and $\mathscr{A}$, respectively, then $\left(\mathscr{A}_{\lambda} u\right)(t)=A_{\lambda} u(t)$ for all $\lambda>0$, a.e. $t \in[0, T]$, for $u \in L^{2}(0, T ; H)$.

Definition 2.1. A sequence $\left\{A^{n}\right\}$ of maximal monotone operators in $H$ is said to be convergent to $A$ in the sense of the resolvent if

$$
\left(I+\lambda A^{n}\right)^{-1} \xi \longrightarrow(I+\lambda A)^{-1} \xi \quad \text { as } n \longrightarrow \infty, \forall \xi \in H, \forall \lambda>0 .
$$


The following characterization of the convergence in the sense of resolvent is true even in reflexive Banach spaces (see [8, page 365]).

Theorem 2.2. If $A: D(A) \subseteq H \rightarrow H$ and $A^{n}: D\left(A^{n}\right) \subseteq H \rightarrow H$ are maximal monotone operators in the Hilbert space $H$, then $A^{n}$ converges to $A$ in the sense of the resolvent if and only if $A^{n}$ is graph-convergent to $A$ (denoted $A^{n} \stackrel{G}{\rightarrow} A$ as $\left.n \rightarrow \infty\right)$, that is, for all $x \in D(A)$, and for all $y \in A x$, there exist $x_{n} \in D\left(A^{n}\right), y_{n} \in A^{n} x_{n}$ such that $x_{n} \rightarrow x, y_{n} \rightarrow y$ in $H$.

We recall now the definition of the Mosco convergence of a sequence of functions and a result concerning the equivalence between the Mosco convergence of the functions $\left(\varphi^{n}\right)$ and the convergence in the sense of the resolvent of the subdifferential operators $\left(\partial \varphi^{n}\right)$ (see [8, Theorem 3.66, page 373]).

Definition 2.3. If $\varphi, \varphi^{n}: H \rightarrow(-\infty, \infty]$ is a sequence of convex, lower-semicontinuous, proper functions, then $\varphi^{n}$ is convergent to $\varphi$ in the sense of Mosco (see $\left.[7,8]\right)$ if

(a) there exists $\left\{x_{n}\right\} \subset H, x_{n} \rightarrow x$, such that $\varphi^{n}\left(x_{n}\right) \rightarrow \varphi(x)$;

(b) for all $\left\{x_{n}\right\} \subset H$ with $x_{n} \rightarrow x$, $\liminf _{n \rightarrow \infty} \varphi^{n}\left(x_{n}\right) \geq \varphi(x)$.

Theorem 2.4. If $\varphi^{n}, \varphi: H \rightarrow(-\infty, \infty]$ are convex, lower-semicontinuous, proper functions, then the following statements are equivalent:

(a) $\varphi^{n} \rightarrow \varphi$ in the sense of Mosco;

(b) $\left(I+\lambda \partial \varphi^{n}\right)^{-1} \xi \rightarrow(I+\lambda \partial \varphi)^{-1} \xi$, for all $\lambda>0$ and for all $\xi \in H$ and there exist $(u, v) \in$ $\partial \varphi$, and $\left(u_{n}, v_{n}\right) \in \partial \varphi^{n}$ such that $u_{n} \rightarrow u, v_{n} \rightarrow v$ in $H$ and $\varphi^{n}\left(u_{n}\right) \rightarrow \varphi(u)$.

\section{The main result}

Consider the boundary value problems

$$
\begin{gathered}
p u^{\prime \prime}+r u^{\prime} \in A u+f, \quad \text { a.e. } t \in(0, T), \\
u(0)=a, \quad u(T)=b, \\
p u_{n}^{\prime \prime}+r u_{n}^{\prime} \in A^{n} u_{n}+f_{n}, \quad \text { a.e. } t \in(0, T), \\
u_{n}(0)=a_{n}, \quad u_{n}(T)=b_{n} .
\end{gathered}
$$

We now state our basic assumptions:

(H1) $A, A^{n}$ are nonlinear (possibly multivalued) maximal monotone operators in the real Hilbert space $H$, with the domains $D(A), D\left(A^{n}\right)$ and $0 \in D(A) \cap D\left(A^{n}\right)$ for all $n \in \mathbb{N}, n \geq 1$;

(H2) $a, b \in D(A), a_{n}, b_{n} \in D\left(A^{n}\right)$ are given elements;

(H3) $f, f_{n} \in L^{2}(0, T ; H)$;

(H4) $p, r:[0, T] \rightarrow \mathbb{R}$ are continuous functions, $p(t) \geq c>0$ for all $t \in[0, T]$.

These hypotheses assure the existence and the uniqueness in $W^{2,2}(0, T ; H)$ of the solutions to problems (3.1)-(3.2) and (3.3)-(3.4), respectively. In addition, suppose that

(H5) if $\left(A^{n}\right)^{0}$ is the minimal section of $A^{n}$, then $\left(A^{n}\right)^{0} a_{n}$ and $\left(A^{n}\right)^{0} b_{n}$ are bounded in $H$;

(H6) $a_{n} \rightarrow a, b_{n} \rightarrow b$ in $H$ and $f_{n} \rightarrow f$ in $L^{2}(0, T ; H)$; 
(H7) $\left(A^{n}\right)$ converges to $A$ in the sense of the resolvent, that is,

$$
\left(I+\lambda A^{n}\right)^{-1} \xi \longrightarrow(I+\lambda A)^{-1} \xi, \quad n \longrightarrow \infty, \forall \xi \in H, \forall \lambda>0
$$

The continuous dependence on data result for the problem (3.1)-(3.2) may now be stated.

THeOrem 3.1. If hypotheses (H1)-(H7) hold and $u, u_{n}$ are the solutions of problems (3.1)(3.2) and (3.3)-(3.4), respectively, then $u_{n}(t) \rightarrow u(t)$ uniformly on $[0, T]$ and $u_{n}^{\prime} \rightarrow u^{\prime}$ in $L^{2}(0, T ; H)$ as $n \rightarrow \infty$.

The proof of this theorem is the purpose of the next section.

Remark 3.2. In [7, Theorem 3.2, page 62 and Proposition 3.7, page 64], the author establishes some conditions when the sum $A^{n}+B^{n}$ converges to $A+B$ in the sense of the resolvent. Here $A^{n}$ and $B^{n}$ are supposed to be maximal monotone operators convergent to $A$ and $B$, respectively, in the sense of the resolvent.

Theorem 3.1 above is not a consequence of these general perturbation results. Indeed, problems (3.1)-(3.2) and (3.3)-(3.4) can be written in $L^{2}(0, T ; H)$ as

$$
-f \in B u+\mathscr{A} u, \quad-f_{n} \in B^{n} u_{n}+\mathscr{A}^{n} u_{n},
$$

respectively, where $\mathscr{A}, \mathscr{A}^{n}$ are the realizations of $A, A^{n}$ in $L^{2}(0, T ; H), B$ is given in (1.14), and $B^{n}$ is analogous to $B$, but with $a_{n}, b_{n}$ instead of $a, b$. It is known that $B, B^{n}$ are maximal monotone in $L^{2}(0, T ; H)$ (see [1]). Moreover, $B=\partial \varphi, B^{n}=\partial \varphi^{n}$, where $\varphi, \varphi^{n}$ : $L^{2}(0, T ; H) \rightarrow(-\infty, \infty]$ are defined by

$$
\begin{gathered}
\varphi(u)= \begin{cases}\frac{1}{2} \int_{0}^{T} \tilde{r}(t)\left\|u^{\prime}(t)\right\|^{2} d t, & u \in W^{1,2}(0, T ; H), u(0)=a, u(T)=b, \\
+\infty, & \text { otherwise, }\end{cases} \\
\varphi^{n}(u)= \begin{cases}\frac{1}{2} \int_{0}^{T} \tilde{r}(t)\left\|u^{\prime}(t)\right\|^{2} d t, & u \in W^{1,2}(0, T ; H), u(0)=a_{n}, u(T)=b_{n}, \\
+\infty, & \text { otherwise. }\end{cases}
\end{gathered}
$$

We show that $\varphi^{n}$ is not Mosco convergent to $\varphi$. To do this, consider $u \in W^{1,2}(0, T ; H)$ with $u(T) \neq b$ and

$$
u_{n}(t)= \begin{cases}a_{n}, & t=0, \\ b_{n}, & t=T, \\ u(t)+\frac{C}{n}, & 0<t<T,\end{cases}
$$

where $C$ is a constant in $H$. It is clear that $u_{n} \rightarrow u$ in $L^{2}(0, T ; H)$ and $\liminf _{n \rightarrow \infty} \varphi^{n}\left(u_{n}\right)<$ $\varphi(u)=+\infty$. Thus condition $(b)$ in Definition 2.3 is not satisfied. Then $\varphi^{n}$ does not converge to $\varphi$ in the sense of Mosco. Theorem 2.4 implies that $\partial \varphi^{n}$ is not convergent to $\partial \varphi$ in the sense of the resolvent. So Attouch's results for the convergence of the sum $\mathscr{A}^{n}+B^{n}$ are not applicable here, even if $\mathscr{A}^{n} \rightarrow \mathscr{A}$ in $L^{2}(0, T ; H)$ in the sense of the resolvent. 
A particular case of Theorem 3.1 is obtained assuming that $A$ and $A^{n}$ are subdifferential mappings and replacing $(H 7)$ by the condition

$(H 7)^{\prime} \varphi^{n} \rightarrow \varphi$ in the sense of Mosco.

In this case, we find (in view of Theorem 2.4) the following consequence of Theorem 3.1 .

Corollary 3.3. If $A=\partial \varphi$ and $A^{n}=\partial \varphi^{n}$, where $\varphi^{n}, \varphi: H \rightarrow(-\infty, \infty]$ are convex, lowersemicontinuous, proper functions with $0 \in D(\partial \varphi), 0 \in D\left(\partial \varphi^{n}\right)$, then under hypotheses $(H 2)-(H 6),(H 7)^{\prime}$, the convergences $u_{n}(t) \rightarrow u(t)$ uniformly on $[0, T]$ and $u_{n}^{\prime} \rightarrow u^{\prime}$ in $L^{2}(0$, $T ; H)$ as $n \rightarrow \infty$ are obtained.

\section{The proof of Theorem 3.1}

The proof of the main result combines some ideas from $[1,2,5]$. For every given $\lambda>0$, we put

$$
\begin{aligned}
& y_{\lambda}=(I+\sqrt{\lambda} A)^{-1} a, \quad z_{\lambda}=(I+\sqrt{\lambda} A)^{-1} b \\
& y_{n \lambda}=\left(I+\sqrt{\lambda} A^{n}\right)^{-1} a, \quad z_{n \lambda}=\left(I+\sqrt{\lambda} A^{n}\right)^{-1} b \text {. }
\end{aligned}
$$

By hypothesis (3.5), it follows that

$$
y_{n \lambda} \longrightarrow y_{\lambda}, \quad z_{n \lambda} \longrightarrow z_{\lambda}, \quad A_{\lambda}^{n} \xi \longrightarrow A_{\lambda} \xi \quad \text { as } n \longrightarrow \infty,
$$

for all $\lambda>0$, and for all $\xi \in H$, where $A_{\lambda}^{n}$ is the Yosida approximation of $A^{n}$.

Let $w_{\lambda}, v_{\lambda}, w_{n \lambda}, v_{n \lambda}$ be the solutions of the auxiliary boundary value problems

$$
\begin{gathered}
p w_{\lambda}^{\prime \prime}+r w_{\lambda}^{\prime} \in A w_{\lambda}+f, \quad \text { a.e. } t \in(0, T), \\
w_{\lambda}(0)=y_{\lambda}, \quad w_{\lambda}(T)=z_{\lambda}, \\
p v_{\lambda}^{\prime \prime}+r v_{\lambda}^{\prime}=A_{\lambda} v_{\lambda}+f, \quad \text { a.e. } t \in(0, T), \\
v_{\lambda}(0)=y_{\lambda}, \quad v_{\lambda}(T)=z_{\lambda}, \\
p w_{n \lambda}^{\prime \prime}+r w_{n \lambda}^{\prime} \in A^{n} w_{n \lambda}+f_{n}, \quad \text { a.e. } t \in(0, T), \\
w_{n \lambda}(0)=y_{n \lambda}, \quad w_{n \lambda}(T)=z_{n \lambda}, \\
p v_{n \lambda}^{\prime \prime}+r v_{n \lambda}^{\prime}=A_{\lambda}^{n} v_{n \lambda}+f_{n}, \quad \text { a.e. } t \in(0, T), \\
v_{n \lambda}(0)=y_{n \lambda}, \quad v_{n \lambda}(T)=z_{n \lambda},
\end{gathered}
$$

respectively. From the general theory recalled in Section 1, we know that each of these problems has a unique solution in $W^{2,2}(0, T ; H)$.

For every $t \in[0, T], \lambda>0$, and $n \in \mathbb{N}$, we can write

$$
\begin{aligned}
\left\|u_{n}(t)-u(t)\right\| \leq & \left\|u_{n}(t)-w_{n \lambda}(t)\right\|+\left\|w_{n \lambda}(t)-v_{n \lambda}(t)\right\| \\
& +\left\|v_{n \lambda}(t)-v_{\lambda}(t)\right\|+\left\|v_{\lambda}(t)-w_{\lambda}(t)\right\| \\
& +\left\|w_{\lambda}(t)-u(t)\right\|, \\
\left|u_{n}^{\prime}-u^{\prime}\right| \leq & \left|u_{n}^{\prime}-w_{n \lambda}^{\prime}\right|+\left|w_{n \lambda}^{\prime}-v_{\nu \lambda}^{\prime}\right|+\left|v_{n \lambda}^{\prime}-v_{\lambda}^{\prime}\right|+\left|v_{\lambda}^{\prime}-w_{\lambda}^{\prime}\right|+\left|w_{\lambda}^{\prime}-u^{\prime}\right| .
\end{aligned}
$$


Recall that $|\cdot|$ denotes the norm in $L^{2}(0, T ; H)$. We intend to take the superior limit as $n \rightarrow \infty$ and then the limit as $\lambda \rightarrow 0$ in both (4.7) and (4.8). In order to do this, we estimate each term in (4.7) and (4.8). One begins with some boundedness results.

LEMmA 4.1. Under the hypotheses of Theorem 3.1, if $w_{n \lambda}$ is the solution of problem (4.5), then for every fixed $\lambda>0$,

$$
\begin{gathered}
\limsup _{n \rightarrow \infty}\left\|w_{n \lambda}^{\prime}(0)\right\| \leq c_{1}\left(\left\|A_{\sqrt{\lambda}} a\right\|+\left\|A_{\sqrt{\lambda}} b\right\|+\left\|y_{\lambda}\right\|+\left\|z_{\lambda}\right\|+1\right), \\
\underset{n \rightarrow \infty}{\limsup }\left\|w_{n \lambda}^{\prime}(T)\right\| \leq c_{2}\left(\left\|A_{\sqrt{\lambda}} a\right\|+\left\|A_{\sqrt{\lambda}} b\right\|+\left\|y_{\lambda}\right\|+\left\|z_{\lambda}\right\|+1\right), \\
\limsup _{n \rightarrow \infty}\left|w_{n \lambda}^{\prime}\right| \leq c_{3}\left(\left\|A_{\sqrt{\lambda}} a\right\|+\left\|A_{\sqrt{\lambda}} b\right\|+\left\|y_{\lambda}\right\|+\left\|z_{\lambda}\right\|+1\right), \\
\limsup _{n \rightarrow \infty}\left|w_{n \lambda}^{\prime \prime}\right| \leq c_{4}\left(\left\|A_{\sqrt{\lambda}} a\right\|+\left\|A_{\sqrt{\lambda}} b\right\|+\left\|y_{\lambda}\right\|+\left\|z_{\lambda}\right\|+1\right), \\
\underset{n \rightarrow \infty}{\limsup }\left|w_{n \lambda}\right|_{C} \leq c_{5}\left(\left\|A_{\sqrt{\lambda}} a\right\|+\left\|A_{\sqrt{\lambda}} b\right\|+\left\|y_{\lambda}\right\|+\left\|z_{\lambda}\right\|+1\right),
\end{gathered}
$$

where $|\cdot|_{C}$ is the norm in $C([0, T] ; H)$, that is, $|u|_{C}=\sup _{t \in[0, T]}\|u(t)\|$.

Proof. One approximates (4.5) by

$$
\begin{gathered}
p w_{n \lambda \mu}^{\prime \prime}+r w_{n \lambda \mu}^{\prime}=A_{\mu}^{n} w_{n \lambda \mu}+\mu w_{n \lambda \mu}+f_{n}, \quad \text { a.e. } t \in(0, T) \\
w_{n \lambda \mu}(0)=y_{n \lambda}, \quad w_{n \lambda \mu}(T)=z_{n \lambda}
\end{gathered}
$$

where $A_{\mu}^{n}=(1 / \mu)\left[I-\left(I+\mu A^{n}\right)^{-1}\right]$. We show that $w_{n \lambda \mu}$ is bounded in $C([0, T] ; H)$ with respect to $\mu$ and $w_{n \lambda \mu} \rightarrow w_{n \lambda}$ in $C([0, T] ; H)$ as $\mu \rightarrow 0$.

One writes the equation in (4.13) as in (1.15), multiply it by $(\tilde{r} / p) w_{n \lambda \mu}$ and integrate over $[0, T]$, to obtain

$$
\begin{aligned}
& \int_{0}^{T}\left(\left(\tilde{r} w_{n \lambda \mu}^{\prime}\right)^{\prime}, w_{n \lambda \mu}\right) d t \\
& \quad=\int_{0}^{T} \frac{\tilde{r}}{p}\left(A_{\mu}^{n} w_{n \lambda \mu}, w_{n \lambda \mu}\right) d t+\mu \int_{0}^{T} \frac{\tilde{r}}{p}\left\|w_{n \lambda \mu}\right\|^{2} d t+\int_{0}^{T} \frac{\tilde{r}}{p}\left(f_{n}, w_{n \lambda \mu}\right) d t .
\end{aligned}
$$

Here and everywhere below, we omit the variable $t$ at the functions under integrals.

Without loss of generality, suppose that $0 \in A^{n} 0$. Otherwise, we replace $A^{n} u$ by $\tilde{A}^{n} u=$ $A^{n} u-\left(A^{n}\right)^{0} 0$ and $f_{n}$ by $\tilde{f}_{n}=f_{n}+\left(A^{n}\right)^{0} 0$. Since $A_{\mu}^{n}$ is monotone, by the above equality and $(H 4)$, we obtain

$$
c\left|w_{n \lambda \mu}^{\prime}\right|^{2}+\mu\left|w_{n \lambda \mu}\right|^{2} \leq \widetilde{r}(T)|| w_{n \lambda \mu}^{\prime}(T)\left\|\cdot|| z_{n \lambda}\right\|+|| w_{n \lambda \mu}^{\prime}(0) \| \cdot|| y_{n \lambda}||+\left|f_{n}\right| \cdot\left|w_{n \lambda \mu}\right| .
$$

Since

$$
w_{n \lambda \mu}(t)=y_{n \lambda}+\int_{0}^{t} w_{n \lambda \mu}^{\prime}(s) d s, \quad t \in[0, T],
$$


we deduce that

$$
\left\|w_{n \lambda \mu}(t)\right\| \leq\left\|y_{n \lambda}\right\|+\sqrt{T}\left(\int_{0}^{T}\left\|w_{n \lambda \mu}^{\prime}\right\|^{2} d s\right)^{1 / 2}, \quad t \in[0, T],
$$

so

$$
\left|w_{n \lambda \mu}\right| \leq C_{1}\left(|| y_{n \lambda}||+\left|w_{n \lambda \mu}^{\prime}\right|\right)
$$

The constant $C_{1}$ and all the constants below are positive and independent of $n, \lambda$, and $\mu$. Using (4.18) in (4.15), we find

$$
\begin{aligned}
c\left|w_{n \lambda \mu}^{\prime}\right|^{2} \leq & \tilde{r}(T)|| w_{n \lambda \mu}^{\prime}(T)\|\cdot\| z_{n \lambda}\|+\| w_{n \lambda \mu}^{\prime}(0)\|\cdot\| y_{n \lambda} \| \\
& +C_{1}\left|f_{n}\right|\left(\left\|y_{n \lambda}\right\|+\left|w_{n \lambda \mu}^{\prime}\right|\right),
\end{aligned}
$$

hence, by virtue of boundedness of $f_{n}$,

$$
\left|w_{n \lambda \mu}^{\prime}\right| \leq C_{2}\left(\left\|y_{n \lambda}\right\|^{1 / 2} \cdot\left\|w_{n \lambda \mu}^{\prime}(0)\right\|^{1 / 2}+\left\|z_{n \lambda}\right\|^{1 / 2} \cdot\left\|w_{n \lambda \mu}^{\prime}(T)\right\|^{1 / 2}+\left\|y_{n \lambda}\right\|^{1 / 2}+1\right) .
$$

We now multiply (4.13) by $w_{n \lambda \mu}^{\prime \prime}$ and integrate from $t=0$ to $t=T$ :

$$
\begin{aligned}
& \int_{0}^{T} p\left\|w_{n \lambda \mu}^{\prime \prime}\right\|^{2} d t+\int_{0}^{T} r\left(w_{n \lambda \mu}^{\prime}, w_{n \lambda \mu}^{\prime \prime}\right) d t \\
& \quad=\int_{0}^{T}\left(A_{\mu}^{n} w_{n \lambda \mu}, w_{n \lambda \mu}^{\prime \prime}\right) d t+\mu \int_{0}^{T}\left(w_{n \lambda \mu}, w_{n \lambda \mu}^{\prime \prime}\right) d t+\int_{0}^{T}\left(f_{n}, w_{n \lambda \mu}^{\prime \prime}\right) d t
\end{aligned}
$$

The functions $x \mapsto A_{\mu}^{n} x$ and $t \mapsto w_{n \lambda \mu}(t)$ are Lipschitz continuous, so the application $t \mapsto A_{\mu}^{n} w_{n \lambda \mu}(t)$ is differentiable a.e. on $[0, T]$. Since $x \mapsto A_{\mu}^{n} x$ is monotone, we also have $\left(\left(A_{\mu}^{n} w_{n \lambda \mu}(t)\right)^{\prime}, w_{n \lambda \mu}^{\prime}(t)\right) \geq 0$ a.e. $t \in(0, T)$. Then,

$$
\left(A_{\mu}^{n} w_{n \lambda \mu}, w_{n \lambda \mu}^{\prime \prime}\right) \leq\left(A_{\mu}^{n} w_{n \lambda \mu}, w_{n \lambda \mu}^{\prime}\right)^{\prime}
$$

On the other hand,

$$
\left(w_{n \lambda \mu}, w_{n \lambda \mu}^{\prime \prime}\right)=\left(w_{n \lambda \mu}, w_{n \lambda \mu}^{\prime}\right)^{\prime}-\left\|w_{n \lambda \mu}^{\prime}\right\|^{2} \leq\left(w_{n \lambda \mu}, w_{n \lambda \mu}^{\prime}\right)^{\prime} .
$$

Now (4.22), (4.23), and (4.21) yield

$$
\begin{aligned}
\int_{0}^{T} p\left\|w_{n \lambda \mu}^{\prime \prime}\right\|^{2} d t \leq & \left(A_{\mu}^{n} z_{n \lambda}, w_{n \lambda \mu}^{\prime}(T)\right)-\left(A_{\mu}^{n} y_{n \lambda}, w_{n \lambda \mu}^{\prime}(0)\right) \\
& +\mu\left(z_{n \lambda}, w_{n \lambda \mu}^{\prime}(T)\right)-\mu\left(y_{n \lambda}, w_{n \lambda \mu}^{\prime}(0)\right) \\
& +\left[\left(\int_{0}^{T}\left\|f_{n}\right\|^{2} d t\right)^{1 / 2}+\left(\int_{0}^{T} r^{2}\left\|w_{n \lambda \mu}^{\prime}\right\|^{2} d t\right)^{1 / 2}\right]\left(\int_{0}^{T}\left\|w_{n \lambda \mu}^{\prime \prime}\right\|^{2} d t\right)^{1 / 2}
\end{aligned}
$$


therefore via $(H 4)$,

$$
\begin{aligned}
c\left(\int_{0}^{T}\left\|w_{n \lambda \mu}^{\prime \prime}\right\|^{2} d t\right)^{1 / 2} \leq & \left(\int_{0}^{T}\left\|f_{n}\right\|^{2} d t\right)^{1 / 2}+\left(\int_{0}^{T} r^{2}\left\|w_{n \lambda \mu}^{\prime}\right\|^{2} d t\right)^{1 / 2} \\
& +\sqrt{c}\left(\left\|A_{\mu}^{n} y_{n \lambda}\right\|^{1 / 2}+\left.\sqrt{\mu}\left\|y_{n \lambda}\right\|\right|^{1 / 2}\right)\left\|w_{n \lambda \mu}^{\prime}(0)\right\|^{1 / 2} \\
& +\sqrt{c}\left(\left\|A_{\mu}^{n} z_{n \lambda}\right\|^{1 / 2}+\sqrt{\mu}\left\|z_{n \lambda}\right\|^{1 / 2}\right)\left\|w_{n \lambda \mu}^{\prime}(T)\right\|^{1 / 2} .
\end{aligned}
$$

Inequality (4.20) leads to

$$
\begin{aligned}
\left(\int_{0}^{T}\left\|w_{n \lambda \mu}^{\prime \prime}\right\|^{2} d t\right)^{1 / 2} \leq & C_{3}+C_{4}\left(\left\|\left(A^{n}\right)^{0} y_{n \lambda}\right\|^{1 / 2}+\left\|y_{n \lambda}\right\|^{1 / 2}\right)\left\|w_{n \lambda \mu}^{\prime}(0)\right\|^{1 / 2} \\
& +C_{5}\left(\left\|\left(A^{n}\right)^{0} z_{n \lambda}\right\|^{1 / 2}+\left\|z_{n \lambda}\right\|^{1 / 2}\right)\left\|w_{n \lambda \mu}^{\prime}(T)\right\|^{1 / 2}+C_{6}\left\|y_{n \lambda}\right\|^{1 / 2}
\end{aligned}
$$

for small $\mu>0$. Observe that

$$
\frac{a-y_{n \lambda}}{\sqrt{\lambda}}=A_{\sqrt{\lambda}}^{n} a \in A^{n} y_{n \lambda}, \quad \frac{b-z_{n \lambda}}{\sqrt{\lambda}}=A_{\sqrt{\lambda}}^{n} b \in A^{n} z_{n \lambda} .
$$

Therefore, we get

$$
\left\|\left(A^{n}\right)^{0} y_{n \lambda}\right\| \leq\left\|A_{\sqrt{\lambda} a}^{n} a, \quad\right\|\left(A^{n}\right)^{0} z_{n \lambda}\|\leq\| A_{\sqrt{\lambda}}^{n} b \| .
$$

So, (4.26) gives

$$
\begin{aligned}
\left(\int_{0}^{T}\left\|w_{n \lambda \mu}^{\prime \prime}\right\|^{2} d t\right)^{1 / 2} \leq & C_{3}+C_{4}\left(\left\|A_{\sqrt{\lambda}}^{n} a\right\|^{1 / 2}+\left\|y_{n \lambda}\right\|^{1 / 2}\right)\left\|w_{n \lambda \mu}^{\prime}(0)\right\|^{1 / 2} \\
& +C_{5}\left(\left\|A_{\sqrt{\lambda}}^{n} b\right\|^{1 / 2}+\left\|z_{n \lambda}\right\|^{1 / 2}\right)\left\|w_{n \lambda \mu}^{\prime}(T)\right\|^{1 / 2}+C_{6}\left\|y_{n \lambda}\right\|^{1 / 2} .
\end{aligned}
$$

To estimate $w_{n \lambda \mu}^{\prime}(0)$ and $w_{n \lambda \mu}^{\prime}(T)$, we write

$$
\begin{aligned}
& w_{n \lambda \mu}^{\prime}(0)=\frac{1}{T}\left[z_{n \lambda}-y_{n \lambda}-\int_{0}^{T}\left(\int_{0}^{t} w_{n \lambda \mu}^{\prime \prime}(s) d s\right) d t\right], \\
& w_{n \lambda \mu}^{\prime}(T)=\frac{1}{T}\left[z_{n \lambda}-y_{n \lambda}+\int_{0}^{T}\left(\int_{t}^{T} w_{n \lambda \mu}^{\prime \prime}(s) d s\right) d t\right] .
\end{aligned}
$$

Now (4.29) and (4.30) imply

$$
\begin{aligned}
\left\|w_{n \lambda \mu}^{\prime}(0)\right\| \leq & C_{7}\left(\left\|y_{n \lambda}\right\|+\left\|z_{n \lambda}\right\|\right)+C_{8}\left(\left\|A_{\sqrt{\lambda}}^{n} a\right\|^{1 / 2}+\left\|y_{n \lambda}\right\|^{1 / 2}\right)\left\|w_{n \lambda \mu}^{\prime}(0)\right\|^{1 / 2} \\
& +C_{9}\left(\left\|A_{\sqrt{\lambda}}^{n} b\right\|^{1 / 2}+\left\|z_{n \lambda}\right\|^{1 / 2}\right)\left\|w_{n \lambda \mu}^{\prime}(T)\right\|^{1 / 2}+C_{10},
\end{aligned}
$$


and analogously for $\left\|w_{n \lambda \mu}^{\prime}(T)\right\|$. Hence,

$$
\begin{aligned}
& \left\|w_{n \lambda \mu}^{\prime}(0)\right\|^{1 / 2} \leq C_{11}\left(\left\|A_{\sqrt{\lambda}}^{n} a\right\|^{1 / 2}+\left\|A_{\sqrt{\lambda}}^{n} b\right\|^{1 / 2}+\left\|y_{n \lambda}\right\|^{1 / 2}+\left\|z_{n \lambda}\right\|^{1 / 2}+1\right), \\
& \left\|w_{n \lambda \mu}^{\prime}(T)\right\|^{1 / 2} \leq C_{12}\left(\left\|A_{\sqrt{\lambda}}^{n} a\right\|^{1 / 2}+\left\|A_{\sqrt{\lambda}}^{n} b\right\|^{1 / 2}+\left\|y_{n \lambda}\right\|^{1 / 2}+\left\|z_{n \lambda}\right\|^{1 / 2}+1\right) .
\end{aligned}
$$

Using (4.32) in (4.29) and (4.20), respectively, we infer that

$$
\begin{aligned}
& \left|w_{n \lambda \mu}^{\prime \prime}\right| \leq C_{13}\left(\left\|A_{\sqrt{\lambda}}^{n} a\right\|+\left\|A_{\sqrt{\lambda}}^{n} b\right\|+\left\|y_{n \lambda}\right\|+\left\|z_{n \lambda}\right\|+1\right), \\
& \left|w_{n \lambda \mu}^{\prime}\right| \leq C_{14}\left(\left\|A_{\sqrt{\lambda}}^{n} a\right\|+\left\|A_{\sqrt{\lambda}}^{n} b\right\|+\left\|y_{n \lambda}\right\|+\left\|z_{n \lambda}\right\|+1\right) .
\end{aligned}
$$

The last inequality, together with (4.17), leads to

$$
\left|w_{n \lambda \mu}\right|_{C} \leq C_{15}\left(\left\|A_{\sqrt{\lambda}}^{n} a\right\|+\left\|A_{\sqrt{\lambda}}^{n} b\right\|+\left\|y_{n \lambda}\right\|+\left\|z_{n \lambda}\right\|+1\right) .
$$

Inequalities (4.32) - (4.34) show that $w_{n \lambda \mu}^{\prime}(0)$ and $w_{n \lambda \mu}^{\prime}(T)$ are bounded in $H, w_{n \lambda \mu}^{\prime \prime}, w_{n \lambda \mu}^{\prime}$ are bounded in $L^{2}(0, T ; H)$, and $w_{n \lambda \mu}$ is bounded in $C([0, T] ; H)$, all of them with respect to $\mu$. By $(4.13)$ we have also the boundedness of $A_{\mu}^{n} w_{n \lambda \mu}$ in $L^{2}(0, T ; H)$ (in $\left.\mu\right)$.

We prove now that $\left(w_{n \lambda \mu}^{\prime}\right)_{\mu}$ is strongly convergent in $C([0, T] ; H)$. To do this, we write (4.13) for $\mu$ and $\nu$, subtract them, multiply by $(\tilde{r} / p)\left(w_{n \lambda \mu}-w_{n \lambda \nu}\right)$, and integrate over $[0, T]$. With the aid of

$$
J_{\mu}^{n} w_{n \lambda \mu}+\mu A_{\mu}^{n} w_{n \lambda \mu}=w_{n \lambda \mu}
$$

one arrives at

$$
\begin{aligned}
-\int_{0}^{T} \tilde{r}\left\|w_{n \lambda \mu}^{\prime}-w_{n \lambda \nu}^{\prime}\right\|^{2} d t= & \int_{0}^{T} \frac{\tilde{r}}{p}\left(A_{\mu}^{n} w_{n \lambda \mu}-A_{\nu}^{n} w_{n \lambda \nu}, J_{\mu}^{n} w_{n \lambda \mu}-J_{\nu}^{n} w_{n \lambda \nu}\right) d t \\
& +\int_{0}^{T} \frac{\tilde{r}}{p}\left(A_{\mu}^{n} w_{n \lambda \mu}-A_{\nu}^{n} w_{n \lambda \nu}, \mu A_{\mu}^{n} w_{n \lambda \mu}-v A_{\nu}^{n} w_{n \lambda \nu}\right) d t \\
& +\int_{0}^{T} \frac{\tilde{r}}{p}\left(\mu w_{n \lambda \mu}-\nu w_{n \lambda \nu}, w_{n \lambda \mu}-w_{n \lambda \nu}\right) d t
\end{aligned}
$$

Since $A_{\mu}^{n} w_{n \lambda \mu} \in A^{n}\left(J_{\mu}^{n} w_{n \lambda \mu}\right)$ and $A^{n}$ is monotone, this implies that

$$
\begin{aligned}
& \int_{0}^{T} \tilde{r}\left\|w_{n \lambda \mu}^{\prime}-w_{n \lambda \nu}^{\prime}\right\|^{2} d t \\
& \quad \leq(\mu+\nu)\left[\int_{0}^{T} \frac{\tilde{r}}{p}\left(A_{\mu}^{n} w_{n \lambda \mu}, A_{\nu}^{n} w_{n \lambda \nu}\right) d t+\int_{0}^{T} \frac{\tilde{r}}{p}\left(w_{n \lambda \mu}, w_{n \lambda \nu}\right) d t\right] .
\end{aligned}
$$

The boundedness in $L^{2}(0, T ; H)$ of $w_{n \lambda \mu}$ and $A_{\mu}^{n} w_{n \lambda \mu}$ with respect to $\mu$ shows that

$$
\left|w_{n \lambda \mu}^{\prime}-w_{n \lambda \nu}^{\prime}\right|^{2} \leq K_{1}^{n \lambda}(\mu+\nu)
$$


where $K_{1}^{n \lambda}$ depends linearly on $\left\|A_{\sqrt{\lambda}}^{n} a\right\|,\left\|A_{\sqrt{\lambda}}^{n} b\right\|,\left\|y_{n \lambda}\right\|,\left\|z_{n \lambda}\right\|$ and is independent of $\mu$ and $\nu$. Consequently, $\left(w_{n \lambda \mu}^{\prime}\right)_{\mu}$ strongly converges in $L^{2}(0, T ; H)$, say

$$
w_{n \lambda \mu}^{\prime} \longrightarrow g_{n \lambda} \quad \text { in } L^{2}(0, T ; H) \text { as } \mu \longrightarrow 0 .
$$

Now we have the estimate

$$
\begin{aligned}
& \left\|w_{n \lambda \mu}^{\prime}(t)-g_{n \lambda}(t)\right\|^{2}-\left\|w_{n \lambda \mu}^{\prime}\left(t_{0}\right)-g_{n \lambda}\left(t_{0}\right)\right\|^{2} \\
& \quad \leq 2\left(\int_{0}^{T}\left\|w_{n \lambda \mu}^{\prime}-g_{n \lambda}\right\|^{2} d t\right)^{1 / 2}\left(\int_{0}^{T}\left\|w_{n \lambda \mu}^{\prime \prime}-g_{n \lambda}^{\prime}\right\|^{2} d t\right)^{1 / 2}
\end{aligned}
$$

with $t_{0} \in[0, T]$ such as $\left\|w_{n \lambda \mu}^{\prime}\left(t_{0}\right)-g_{n \lambda}\left(t_{0}\right)\right\| \rightarrow 0$ as $\mu \rightarrow 0$. Since $\left(w_{n \lambda \mu}^{\prime \prime}\right)_{\mu}$ is bounded in $L^{2}(0, T ; H)$ with respect to $\mu$, one deduces the convergence

$$
w_{n \lambda \mu}^{\prime} \longrightarrow g_{n \lambda} \quad \text { in } C([0, T] ; H) \text { as } \mu \longrightarrow 0 .
$$

From (4.16) and (4.41), we obtain that

$$
w_{n \lambda \mu}(t) \longrightarrow y_{n \lambda}+\int_{0}^{t} g_{n \lambda}(s) d s \stackrel{\text { not. }}{=} h_{n \lambda}(t), \quad t \in[0, T] .
$$

It follows that $h_{n \lambda}(0)=y_{n \lambda}$ and $h_{n \lambda}$ is differentiable on $[0, T]$ with $h_{n \lambda}^{\prime}=g_{n \lambda}$. Hence, $w_{n \lambda \mu}^{\prime} \rightarrow h_{n \lambda}^{\prime}$ in $C([0, T] ; H), w_{n \lambda \mu} \rightarrow h_{n \lambda}, w_{n \lambda \mu}^{\prime \prime} \rightarrow h_{n \lambda}^{\prime \prime}$ in $L^{2}(0, T ; H)$, and $h_{n \lambda}(T)=z_{n \lambda}$. By (4.35) we get

$$
J_{\mu}^{n} w_{n \lambda \mu}-h_{n \lambda} \quad \text { as } \mu \longrightarrow 0 \text { in } L^{2}(0, T ; H) .
$$

Denoting by $B_{1}$ the operator

$$
\begin{gathered}
B_{1} u=-p u^{\prime \prime}-r u^{\prime}=-\frac{p}{\widetilde{r}}\left(\tilde{r} u^{\prime}\right)^{\prime}, \\
D\left(B_{1}\right)=\left\{u \in W^{2,2}(0, T ; H), u(0)=y_{n \lambda}, u(T)=z_{n \lambda}\right\},
\end{gathered}
$$

we may write (4.13) under the form

$$
-B_{1} w_{n \lambda \mu}-\mu w_{n \lambda \mu}-f_{n} \in \mathscr{A}^{n}\left(\mathscr{F}_{\mu}^{n} w_{n \lambda \mu}\right),
$$

where $\mathscr{F}_{\mu}^{n}$ is the realization of $J_{\mu}^{n}$ in $L^{2}(0, T ; H),\left(\mathscr{F}_{\mu}^{n} u\right)(t)=J_{\mu}^{n} u(t)$ a.e. $t \in[0, T]$ for $u \in$ $L^{2}(0, T ; H)$.

Observe that $-B_{1} w_{n \lambda \mu}-\mu w_{n \lambda \mu}-f_{n}--B_{1} h_{n \lambda}-f_{n}$. Taking into account the maximal monotonicity of $\mathscr{A}^{n}$ in $L^{2}(0, T ; H)$ and (4.43), in order to take the limit in (4.45), it is enough to prove that

$$
\left\langle-B_{1} w_{n \lambda \mu}-\mu w_{n \lambda \mu}-f_{n}, \Phi_{\mu}^{n} w_{n \lambda \mu}\right\rangle \longrightarrow\left\langle-B_{1} h_{n \lambda}-f_{n}, h_{n \lambda}\right\rangle .
$$

Using (4.35) and the boundedness of $B_{1} w_{n \lambda \mu}, \mathscr{A}^{n} w_{n \lambda \mu}, w_{n \lambda \mu}$, and $\mathscr{f}_{\mu}^{n} w_{n \lambda \mu}$, it suffices to show the convergence

$$
\left\langle-B_{1} w_{n \lambda \mu}-f_{n}, w_{n \lambda \mu}\right\rangle \longrightarrow\left\langle-B_{1} h_{n \lambda}-f_{n}, h_{n \lambda}\right\rangle .
$$


But $\left\langle f_{n}, w_{n \lambda \mu}\right\rangle \rightarrow\left\langle f_{n}, h_{n \lambda}\right\rangle$ and by virtue of (4.41) we get

$$
\begin{aligned}
\left\langle-B_{1} w_{n \lambda \mu}, w_{n \lambda \mu}\right\rangle & =\tilde{r}(T)\left(w_{n \lambda \mu}^{\prime}(T), z_{n \lambda}\right)-\left(w_{n \lambda \mu}^{\prime}(0), y_{n \lambda}\right)-\int_{0}^{T} \tilde{r}\left\|w_{n \lambda \mu}^{\prime}\right\|^{2} d t \\
& \longrightarrow \tilde{r}(T)\left(h_{n \lambda}^{\prime}(T), z_{n \lambda}\right)-\left(h_{n \lambda}^{\prime}(0), y_{n \lambda}\right)-\int_{0}^{T} \tilde{r}\left\|h_{n \lambda}^{\prime}\right\|^{2} d t \\
& =\left\langle-B_{1} h_{n \lambda}, h_{n \lambda}\right\rangle .
\end{aligned}
$$

Thus (4.46) is proved. Now we may pass to the limit as $\mu \rightarrow 0$ in (4.45) and find that $h_{n \lambda} \in D\left(A^{n}\right)$ and $-B_{1} h_{n \lambda}-f_{n} \in \mathscr{A}^{n} h_{n \lambda}$. Since $w_{n \lambda}$ verifies the same equation, by the uniqueness one deduces $h_{n \lambda}=w_{n \lambda}$. Therefore, $w_{n \lambda \mu} \rightarrow w_{n \lambda}, w_{n \lambda \mu}^{\prime \prime} \rightarrow w_{n \lambda}^{\prime \prime}$ in $L^{2}(0, T ; H)$, $w_{n \lambda \mu}^{\prime} \rightarrow w_{n \lambda}^{\prime}$ in $C([0, T] ; H), w_{n \lambda \mu}(t) \rightarrow w_{n \lambda}(t)$ for all $t \in[0, T]$. Now (4.9)-(4.12) follow from (4.32)-(4.34). The proof is finished.

We now give a boundedness result for the solution $\left(u_{n}\right)$ of (3.4)-(3.5).

LemmA 4.2. If the hypotheses of Theorem 3.1 are satisfied, then $\left\{u_{n}^{\prime}(0)\right\}$ and $\left\{u_{n}^{\prime}(T)\right\}$ are bounded in $H,\left\{u_{n}^{\prime}\right\},\left\{u_{n}^{\prime \prime}\right\}$ are bounded in $L^{2}(0, T ; H)$, and $\left\{u_{n}\right\}$ is bounded in $C([0, T] ; H)$.

Proof. Consider the boundary value problem

$$
\begin{gathered}
p u_{n \mu}^{\prime \prime}+r u_{n \mu}^{\prime}=A_{\mu}^{n} u_{n \mu}+\mu u_{n \mu}+f_{n}, \quad \text { a.e. } t \in(0, T), \\
u_{n \mu}(0)=a_{n}, \quad u_{n \mu}(T)=b_{n} .
\end{gathered}
$$

Following the computation from the proof of Lemma 4.1, we get an estimate of the form (4.20) with $a_{n}, b_{n}$ instead of $y_{n \lambda}, z_{n \lambda}$. Since $\left(a_{n}\right),\left(b_{n}\right)$ are bounded, this can be written as

$$
\left|u_{n \mu}^{\prime}\right| \leq k_{1}\left\|u_{n \mu}^{\prime}(0)\right\|^{1 / 2}+k_{2}\left\|u_{n \mu}^{\prime}(T)\right\|^{1 / 2}+k_{3}
$$

where $k_{1}, k_{2}, k_{3}>0$ are independent of $n$ and $\mu$.

Similarly, one obtains an inequality of the form (4.26), namely,

$$
\begin{aligned}
\left|u_{n \mu}^{\prime \prime}\right| \leq & k_{4}+k_{5}\left(\left\|\left(A^{n}\right)^{0} a_{n}\right\|^{1 / 2}+\left\|a_{n}\right\|^{1 / 2}\right)\left\|u_{n \mu}^{\prime}(0)\right\|^{1 / 2} \\
& +k_{6}\left(\left\|\left(A^{n}\right)^{0} b_{n}\right\|^{1 / 2}+\left\|b_{n}\right\|^{1 / 2}\right)\left\|u_{n \mu}^{\prime}(T)\right\|^{1 / 2}+k_{7}\left\|a_{n}\right\|^{1 / 2} .
\end{aligned}
$$

Hypotheses (H5) and (H6) imply the existence of some constants $k_{8}, k_{9}, k_{10}>0$ (independent of $n$ and $\mu$ ) such that

$$
\left|u_{n \mu}^{\prime \prime}\right| \leq k_{8}\left\|u_{n \mu}^{\prime}(0)\right\|^{1 / 2}+k_{9}\left\|u_{n \mu}^{\prime}(T)\right\|^{1 / 2}+k_{10} .
$$

Next, as in (4.31), one arrives at

$$
\left\|u_{n \mu}^{\prime}(0)\right\| \leq k_{11}\left\|u_{n \mu}^{\prime}(0)\right\|^{1 / 2}+k_{12}\left\|u_{n \mu}^{\prime}(T)\right\|^{1 / 2}+k_{13},
$$

and an analogous inequality for $\left\|u_{n \mu}^{\prime}(T)\right\|$, with all constants independent of $n$ and $\mu$. This provides upper bounds for $\left\|u_{n \mu}^{\prime}(0)\right\|,\left\|u_{n \mu}^{\prime}(T)\right\|$ and via (4.50), (4.52), for $\left|u_{n \mu}^{\prime}\right|,\left|u_{n \mu}^{\prime \prime}\right|$. By $u_{n \mu}(t)=a_{n}+\int_{0}^{T} u_{n \mu}^{\prime}(s) d s, t \in[0, T]$, we find an upper bound for $u_{n \mu}$ in $C([0, T] ; H)$. 
Now, as in the proof of the previous lemma, one shows that $u_{n \mu} \rightarrow u_{n}, u_{n \mu}^{\prime \prime} \rightarrow u_{n}^{\prime \prime}$ in $L^{2}(0, T ; H), u_{n \mu}^{\prime} \rightarrow u_{n}^{\prime}$ in $C([0, T] ; H)$, and $u_{n \mu}(t) \rightarrow u_{n}(t)$ for $t \in[0, T]($ as $\mu \rightarrow 0)$.

Since $\left\|u_{n}^{\prime}(0)\right\| \leq \liminf _{\mu \rightarrow 0}\left\|u_{n \mu}^{\prime}(0)\right\|$ and $\left\|u_{n}^{\prime}(T)\right\| \leq \liminf _{\mu \rightarrow 0}\left\|u_{n \mu}^{\prime}(T)\right\|$, one deduces that $u_{n}^{\prime}(0)$ and $u_{n}^{\prime}(T)$ are bounded in $H$. Analogously, $u_{n}^{\prime}, u_{n}^{\prime \prime}$ are bounded in $L^{2}(0, T ; H)$ and $u_{n}$ in $C([0, T] ; H)$, as claimed.

Using the same method we can state that the solution $\left(v_{n \lambda}\right)$ of (4.6) is bounded with respect to $n$ for any fixed $\lambda>0$. Since (4.6) already contains the Yosida approximation $A_{\lambda}^{n}$ of $A^{n}$, we avoid the new parameter $\mu$ and work directly with (4.6). One obtains estimates similar to (4.20), (4.29), and (4.32), where $\left\|y_{n \lambda}\right\|,\left\|z_{n \lambda}\right\|,\left\|A_{\sqrt{\lambda}}^{n} a\right\|,\left\|A_{\sqrt{\lambda}}^{n} b\right\|$ are bounded with respect to $n$, for every given $\lambda>0$. Indeed, by (4.2) we have the convergences $y_{n \lambda} \rightarrow$ $y_{\lambda}, z_{n \lambda} \rightarrow z_{\lambda}, A_{\sqrt{\lambda}}^{n} a \rightarrow A_{\sqrt{\lambda}} a, A_{\sqrt{\lambda}}^{n} b \rightarrow A_{\sqrt{\lambda}} b$ as $n \rightarrow \infty$ for every $\lambda>0$, therefore

$$
\begin{aligned}
\sup _{n \in \mathbb{N}}\left\|y_{n \lambda}\right\|=B_{\lambda}<\infty, & & \sup _{n \in \mathbb{N}}\left\|z_{n \lambda}\right\|=C_{\lambda}<\infty, \\
\sup _{n \in \mathbb{N}}\left\|A_{\sqrt{\lambda}}^{n} a\right\|=D_{\lambda}<\infty, & & \sup _{n \in \mathbb{N}}\left\|A_{\sqrt{\lambda}}^{n} b\right\|=E_{\lambda}<\infty .
\end{aligned}
$$

These lead to the following result.

Lemma 4.3. For every fixed $\lambda>0, v_{n \lambda}^{\prime}(0), v_{n \lambda}^{\prime}(T)$ are bounded in $H$ with respect to $n,\left\{v_{n \lambda}^{\prime}\right\}$, $\left\{v_{n \lambda}^{\prime \prime}\right\}$ are bounded in $L^{2}(0, T ; H)$ and $\left\{v_{n \lambda}\right\}$ is bounded in $C([0, T] ; H)$.

Repeating the proof of Lemma 4.1 for problem (4.3), we get the following.

Lemma 4.4. The solution $w_{\lambda}$ of $(4.3)$ is bounded in $C([0, T] ; H), w_{\lambda}^{\prime}, w_{\lambda}^{\prime \prime}$ are bounded in $L^{2}(0, T ; H)$ and $w_{\lambda}^{\prime}(0), w_{\lambda}^{\prime}(T)$ are bounded in $H$.

Now we are going to estimate each term in (4.7) and (4.8). We begin with the following lemma.

Lemma 4.5. Under the hypotheses of Theorem 3.1, for every given $\lambda>0$,

$$
\begin{aligned}
\limsup _{n \rightarrow \infty}\left|u_{n}^{\prime}-w_{n \lambda}^{\prime}\right| \leq c_{6}( & \left\|A_{\sqrt{\lambda}} a\right\|^{1 / 2}+\left\|A_{\sqrt{\lambda}} b\right\|^{1 / 2}+\left\|y_{\lambda}\right\|^{1 / 2} \\
& \left.+\left\|z_{\lambda}\right\|^{1 / 2}+1\right)\left(\left\|a-y_{\lambda}\right\|^{1 / 2}+\left\|b-z_{\lambda}\right\|^{1 / 2}\right), \\
\limsup _{n \rightarrow \infty}\left|u_{n}-w_{n \lambda}\right|_{C} \leq c_{7}( & \left\|A_{\sqrt{\lambda}} a\right\|^{1 / 2}+\left\|A_{\sqrt{\lambda}} b\right\|^{1 / 2}+\left\|y_{\lambda}\right\|^{1 / 2} \\
& \left.+\left\|z_{\lambda}\right\|^{1 / 2}+1\right)\left(\left\|a-y_{\lambda}\right\|^{1 / 2}+\left\|b-z_{\lambda}\right\|^{1 / 2}\right)+\left\|a-y_{\lambda}\right\| .
\end{aligned}
$$

Proof. Subtracting (3.3) and the equation from (4.5), multiplying by $(\tilde{r} / p)\left(u_{n}-w_{n \lambda}\right)$, and integrating over $[0, T]$ by parts, we get via the monotonicity of $A^{n}$,

$$
\tilde{r}(T)\left(u_{n}^{\prime}(T)-w_{n \lambda}^{\prime}(T), b_{n}-z_{n \lambda}\right)-\left(u_{n}^{\prime}(0)-w_{n \lambda}^{\prime}(0), a_{n}-y_{n \lambda}\right) \geq \int_{0}^{T} \tilde{r}\left\|u_{n}^{\prime}-w_{n \lambda}^{\prime}\right\|^{2} d t
$$


so

$$
\begin{aligned}
c\left|u_{n}^{\prime}-w_{n \lambda}^{\prime}\right|^{2} \leq & \tilde{r}(T)\left\|u_{n}^{\prime}(T)-w_{n \lambda}^{\prime}(T)\right\| \cdot\left\|b_{n}-z_{n \lambda}\right\| \\
& +\left\|u_{n}^{\prime}(0)-w_{n \lambda}^{\prime}(0)\right\| \cdot\left\|a_{n}-y_{n \lambda}\right\| .
\end{aligned}
$$

According to (4.9) and Lemma 4.2, this yields (4.55). Next, from

$$
u_{n}(t)-w_{n \lambda}(t)=a_{n}-y_{n \lambda}+\int_{0}^{T}\left(u_{n}^{\prime}-w_{n \lambda}^{\prime}\right)(s) d s, \quad t \in[0, T]
$$

and (4.55), we derive (4.56).

For the second terms in (4.7) and (4.8), we can also find upper bounds with the aid of $y_{\lambda}, z_{\lambda}, A_{\sqrt{\lambda}} a, A_{\sqrt{\lambda}} b$.

Lemma 4.6. Suppose that the above hypotheses hold and let $w_{n \lambda}, v_{n \lambda}$ be the solutions of boundary value problems (4.5) and (4.6), respectively. Then

$$
\begin{gathered}
\limsup _{n \rightarrow \infty}\left|w_{n \lambda}^{\prime}-v_{n \lambda}^{\prime}\right| \leq c_{8} \sqrt{\lambda}\left(\left\|A_{\sqrt{\lambda}} a\right\|+\left\|A_{\sqrt{\lambda}} b\right\|+\left\|y_{\lambda}\right\|+\left\|z_{\lambda}\right\|+1\right), \\
\limsup _{n \rightarrow \infty}\left|w_{n \lambda}-v_{n \lambda}\right|_{C} \leq c_{8} \sqrt{T \lambda}\left(\left\|A_{\sqrt{\lambda}} a\right\|+\left\|A_{\sqrt{\lambda}} b\right\|+\left\|y_{\lambda}\right\|+\left\|z_{\lambda}\right\|+1\right) .
\end{gathered}
$$

Proof. One subtracts (4.5) and (4.6), multiplies by $(\tilde{r} / p)\left(w_{n \lambda}-v_{n \lambda}\right)$, and integrates from $t=0$ to $t=T$ to obtain

$$
\int_{0}^{T}\left(\left(\tilde{r} w_{n \lambda}^{\prime}-\tilde{r} v_{n \lambda}^{\prime}\right)^{\prime}, w_{n \lambda}-v_{n \lambda}\right) d t=\int_{0}^{T} \frac{\tilde{r}}{p}\left(\alpha_{n \lambda}-A_{\lambda}^{n} v_{n \lambda}, w_{n \lambda}-v_{n \lambda}\right) d t
$$

Here we have denoted for simplicity by $\alpha_{n \lambda}$ the element $p w_{n \lambda}^{\prime \prime}+r w_{n \lambda}^{\prime}-f_{n} \in A^{n} w_{n \lambda}$. Integrating by parts and writing $v_{n \lambda}$ in the right-hand side as $v_{n \lambda}=J_{\lambda}^{n} v_{n \lambda}+\lambda A_{\lambda}^{n} v_{n \lambda}$, we obtain via the monotonicity of $A^{n}$,

$$
\int_{0}^{T} \tilde{r}\left\|w_{n \lambda}^{\prime}-v_{n \lambda}^{\prime}\right\|^{2} d t \leq \lambda \int_{0}^{T} \frac{\tilde{r}}{p}\left(\alpha_{n \lambda}-A_{\lambda}^{n} v_{n \lambda}, A_{\lambda}^{n} w_{n \lambda}\right) d t
$$

This, together with $\left(\alpha_{n \lambda}-A_{\lambda}^{n} v_{n \lambda}, A_{\lambda}^{n} v_{n \lambda}\right) \leq(1 / 2)\left\|\alpha_{n \lambda}\right\|^{2}$, implies

$$
\int_{0}^{T} \tilde{r}\left\|w_{n \lambda}^{\prime}-v_{n \lambda}^{\prime}\right\|^{2} d t \leq C \lambda\left(1+\left|w_{n \lambda}^{\prime \prime}\right|^{2}+\left|w_{n \lambda}^{\prime}\right|^{2}\right)
$$

and in view of (4.10) and (4.11), we arrive at (4.60). With the aid of the equality $w_{n \lambda}(t)-$ $v_{n \lambda}(t)=\int_{0}^{t}\left(w_{n \lambda}^{\prime}-v_{n \lambda}^{\prime}\right)(s) d s, t \in[0, T]$, we can see that $(4.61)$ is also verified.

Analogously with Lemmas 4.5 and 4.6, we derive the following results. 
LEMma 4.7. If $u$ and $w_{\lambda}$ are the solutions of the boundary value problems (3.1)-(3.2) and (4.3), then for every $\lambda>0$,

$$
\begin{aligned}
& \left|u^{\prime}-w_{\lambda}^{\prime}\right| \leq c_{9}\left(\left\|a-y_{\lambda}\right\|^{1 / 2}+\left\|b-z_{\lambda}\right\|^{1 / 2}\right) \\
& \left|u-w_{\lambda}\right|_{C} \leq\left\|a-y_{\lambda}\right\|+c_{10}\left(\left\|a-y_{\lambda}\right\|^{1 / 2}+\left\|b-z_{\lambda}\right\|^{1 / 2}\right)
\end{aligned}
$$

with $c_{9}, c_{10}$ being positive constants.

LEMmA 4.8. If $w_{\lambda}$ and $v_{\lambda}$ are the solutions of (4.3) and (4.4), then

$$
\left|w_{\lambda}^{\prime}-v_{\lambda}^{\prime}\right| \leq c_{11} \sqrt{\lambda}, \quad\left|w_{\lambda}-v_{\lambda}\right|_{C} \leq c_{12} \sqrt{\lambda}
$$

Finally, it will be established that $v_{n \lambda}^{\prime}-v_{\lambda}^{\prime}$ and $v_{n \lambda}-v_{\lambda}$ tend to 0 as $n \rightarrow \infty$, for all $\lambda>0$, in $L^{2}(0, T ; H)$ and in $C([0, T] ; H)$, respectively.

Lemma 4.9. Suppose the assumptions of Theorem 3.1 are satisfied. Then, for every $\lambda>0$,

$$
\lim _{n \rightarrow \infty}\left|v_{n \lambda}^{\prime}-v_{\lambda}^{\prime}\right|=0, \quad \lim _{n \rightarrow \infty}\left|v_{n \lambda}-v_{\lambda}\right|_{C}=0 .
$$

Proof. Subtract (4.6) and (4.4), multiply by $(\tilde{r} / p)\left(v_{n \lambda}-v_{\lambda}\right)$, and integrate over $[0, T]$, deducing thus the equality

$$
\begin{aligned}
\tilde{r}(T)\left(v_{n \lambda}^{\prime}(T)-v_{\lambda}^{\prime}(T), z_{n \lambda}-z_{\lambda}\right)-\left(v_{n \lambda}^{\prime}(0)-v_{\lambda}^{\prime}(0), y_{n \lambda}-y_{\lambda}\right)-\int_{0}^{T} \tilde{r}\left\|v_{n \lambda}^{\prime}-v_{\lambda}^{\prime}\right\|^{2} d t \\
=\int_{0}^{T} \frac{\tilde{r}}{p}\left(A_{\lambda}^{n} v_{n \lambda}-A_{\lambda}^{n} v_{\lambda}, v_{n \lambda}-v_{\lambda}\right) d t+\int_{0}^{T} \frac{\tilde{r}}{p}\left(A_{\lambda}^{n} v_{\lambda}-A_{\lambda} v_{\lambda}, v_{n \lambda}-v_{\lambda}\right) d t \\
\quad+\int_{0}^{T} \frac{\tilde{r}}{p}\left(f_{n}-f, v_{n \lambda}-v_{\lambda}\right) d t
\end{aligned}
$$

or, in view of the monotonicity of $A_{\lambda}^{n}$,

$$
\begin{aligned}
c\left|v_{n \lambda}^{\prime}-v_{\lambda}^{\prime}\right|^{2} \leq & \tilde{r}(T)\left\|v_{n \lambda}^{\prime}(T)-v_{\lambda}^{\prime}(T)\right\| \cdot\left\|z_{n \lambda}-z_{\lambda}\right\|+\left\|v_{n \lambda}^{\prime}(0)-v_{\lambda}^{\prime}(0)\right\| \cdot\left\|y_{n \lambda}-y_{\lambda}\right\| \\
& +\left[\left(\int_{0}^{T} \frac{\tilde{r}}{p}\left\|A_{\lambda}^{n} v_{\lambda}-A_{\lambda} v_{\lambda}\right\|^{2} d t\right)^{1 / 2}+\left(\int_{0}^{T} \frac{\tilde{r}}{p}\left\|f_{n}-f\right\|^{2} d t\right)^{1 / 2}\right] \\
& \times\left(\int_{0}^{T} \frac{\tilde{r}}{p}\left\|v_{n \lambda}-v_{\lambda}\right\|^{2} d t\right)^{1 / 2} .
\end{aligned}
$$

According to the boundedness from Lemma 4.3, this leads to

$$
\left|v_{n \lambda}^{\prime}-v_{\lambda}^{\prime}\right|^{2} \leq k_{\lambda}\left(|| y_{n \lambda}-y_{\lambda}||+|| z_{n \lambda}-z_{\lambda}||+\left|A_{\lambda}^{n} v_{\lambda}-A_{\lambda} v_{\lambda}\right|+\left|f_{n}-f\right|\right)
$$

for all $\lambda>0, n \in \mathbb{N}$, where $k_{\lambda}$ is independent of $n$. By (4.2) we infer that

$$
A_{\lambda}^{n} v_{\lambda}(t) \longrightarrow A_{\lambda} v_{\lambda}(t) \quad \text { uniformly on }[0, T] \text {, as } n \longrightarrow \infty \text {. }
$$


Using this, together with (H6) and the other convergences from (4.2) into (4.70), we find the first part of (4.67). The second limit is immediate.

The end of the proof of Theorem 3.1. We come back to (4.7) and (4.8) and apply Lemmas 4.5-4.9. Therefore, for small $\lambda>0$,

$$
\begin{aligned}
\limsup _{n \rightarrow \infty} & \left|u_{n}-u\right|_{C} \\
\leq & c_{13}\left(\left\|A_{\sqrt{\lambda}} a\right\|^{1 / 2}+\left\|A_{\sqrt{\lambda}} b\right\|^{1 / 2}+\left\|y_{\lambda}\right\|^{1 / 2}+\left\|z_{\lambda}\right\|^{1 / 2}+1\right)\left(\left\|a-y_{\lambda}\right\|^{1 / 2}+\left\|b-z_{\lambda}\right\|^{1 / 2}\right) \\
& +2\left\|a-y_{\lambda}\right\|+c_{8} \sqrt{T \lambda}\left(\left\|A_{\sqrt{\lambda}} a\right\|+\left\|A_{\sqrt{\lambda}} b\right\|+\left\|y_{\lambda}\right\|+\left\|z_{\lambda}\right\|+1\right)+c_{12} \sqrt{\lambda},
\end{aligned}
$$

where $c_{13}>0$ is independent of $\lambda$. A similar inequality is available for limsup $\operatorname{sum}_{n \rightarrow \infty}\left|u_{n}^{\prime}-u^{\prime}\right|$.

Taking into account the boundedness of $A_{\sqrt{\lambda}} a$ and $A_{\sqrt{\lambda}} b$ and the convergences $y_{\lambda} \rightarrow$ $a, z_{\lambda} \rightarrow b$, we may pass to the limit as $\lambda \rightarrow 0$ in the above inequality and conclude that $u_{n}(t) \rightarrow u(t)$ as $n \rightarrow \infty$, uniformly on $[0, T]$. Analogously, $u_{n}^{\prime} \rightarrow u^{\prime}$ in $L^{2}(0, T ; H)$ and the proof is complete.

\section{Internal approximations}

In this section, we give a numerical approximation of the solution $u$ of the problem

$$
\begin{gathered}
p u^{\prime \prime}(t)+r u^{\prime}(t)=A u(t), \quad 0<t<T, \\
u(0)=a, \quad u(T)=b,
\end{gathered}
$$

by the solution $u_{N}$ of an internal scheme of approximation.

Suppose that $H$ is a separable real Hilbert space, provided with the scalar product $(\cdot, \cdot)$ and the corresponding norm $\|\cdot\|$ and

$$
p, r:[0, T] \longrightarrow \mathbb{R} \quad \text { are continuous, } \quad p(t) \geq c>0 \quad \forall t \in[0, T] .
$$

Consider the univoque operator $A: H \rightarrow H$ satisfying the following assumption:

(H8) $A$ is monotone, hemicontinuous, and everywhere defined on $H$.

Then $A$ is maximal monotone in $H$ (see [13, page 40]), and therefore for all $a, b \in H$, problem (5.1) has a unique solution $u \in W^{2,2}(0, T ; H)$ (see [1]).

Let $\left\{e_{i}\right\}_{i=1}^{\infty}$ be an orthonormal basis in $H$. For any fixed positive integer $N$, denote by $P_{N}$ the orthogonal projector given by $P_{N} x=\sum_{i=1}^{N}\left(x, e_{i}\right) e_{i}$ for all $x \in H$ and let $H_{N}=P_{N} H$. It is known that $P_{N}^{2}=P_{N}$ and $P_{N}$ is selfadjoint, that is, $\left(P_{N} x, y\right)=\left(x, P_{N} y\right)$ for all $x, y \in H$ (see, e.g., [17]).

One defines the operator $A_{N}: D\left(A_{N}\right)=H_{N} \subset H \rightarrow H, A_{N}=P_{N} A$. So, for every $\tilde{x}_{N}=$ $P_{N} x \in H_{N}$ (with $x \in H$ ), we have $A_{N} \tilde{x}_{N} \in H_{N}$ and

$$
A_{N} \tilde{x}_{N}=P_{N} A P_{N} x=P_{N} A\left(\sum_{i=1}^{N}\left(x, e_{i}\right) e_{i}\right)
$$


It is easy to check that, in view of $(H 8)$, the operator $A_{N}$ is monotone, hemicontinuous, univoque, and everywhere defined on $H_{N}$. Consequently, it is maximal monotone in $H_{N}$.

Consider now the approximating problem

$$
\begin{gathered}
p u_{N}^{\prime \prime}(t)+r u_{N}^{\prime}(t)=A_{N} u_{N}(t), \quad 0<t<T, \\
u_{N}(0)=P_{N} a, \quad u_{N}(T)=P_{N} b .
\end{gathered}
$$

It is clear that (5.4) has a unique solution $u_{N} \in W^{2,2}\left(0, T ; H_{N}\right)$.

Assume in addition that $A 0=0$ and $A$ is bounded, that is, it maps bounded sets onto bounded sets.

We now show that

$$
\left(I+\lambda A_{N}\right)^{-1} P_{N} x \longrightarrow(I+\lambda A)^{-1} x \quad(N \longrightarrow \infty), \forall \lambda>0, \forall x \in H .
$$

To do this, we put $y_{N}=\left(I+\lambda A_{N}\right)^{-1} P_{N} x$ and $y=(I+\lambda A)^{-1} x$. Therefore, we get $y_{N} \in H_{N}$ and

$$
y_{N}-P_{N} y_{N}+\lambda\left(P_{N} A y_{N}-P_{N} A y\right)=0
$$

Multiplying by $y_{N}-P_{N} y$ in $H$, we obtain $\left\|y_{N}-P_{N} y\right\|^{2}+\lambda\left(P_{N} A y_{N}-P_{N} A y, y_{N}-P_{N} y\right)=0$. Since $P_{N}$ is selfadjoint, $P_{N}^{2}=P_{N}$, and $P_{N} y_{N}=y_{N}$, one deduces that

$$
\left\|y_{N}-P_{N} y\right\|^{2}+\lambda\left(A y_{N}-A y, y_{N}-y\right)+\lambda\left(A y_{N}-A y, y-P_{N} y\right)=0
$$

The sequence $\left\{y_{N}\right\}$ is bounded in $H$ for every fixed $\lambda>0$. Indeed, since $A 0=0$ and $\left(I+\lambda A_{N}\right)^{-1}$ is a contraction, it follows that

$$
\left\|y_{N}\right\|=\left\|\left(I+\lambda A_{N}\right)^{-1} P_{N} x-\left(I+\lambda A_{N}\right)^{-1} 0\right\| \leq\left\|P_{N} x\right\|
$$

Hence, $\left\{y_{N}\right\}$ is bounded in $H$.

Passing to the superior limit as $N \rightarrow \infty$ in (5.7) and using the monotonicity and the boundedness of $A$, we find that $y_{N} \rightarrow y$ in $H$ as $N \rightarrow \infty$, that is, (5.5) holds.

Using again the boundedness of $A$ and that fact that $P_{N}$ is selfadjoint with $P_{N}^{2}=P_{N}$, we can easily show that $A_{N} P_{N} a$ and $A_{N} P_{N} b$ are bounded in $H$. Thus condition (H5) is verified.

As a consequence of Theorem 3.1, we state the following internal approximating result.

Proposition 5.1. Assume that (5.2) holds, $A: H \rightarrow H$ is a bounded operator satisfying (H8), $A 0=0$ and $a, b \in H$ are given. Denoting by $u$ and $u_{N}$ the unique solutions of the boundary values problems (5.1) and (5.4), respectively, where $A_{N}=P_{N} A: H_{N} \rightarrow H_{N}$, the convergences $u_{N}(t) \rightarrow u(t)$ uniformly on $[0, T]$ and $u_{N}^{\prime} \rightarrow u^{\prime}$ in $L^{2}(0, T ; H)$ as $N \rightarrow \infty$ are obtained. 


\section{References}

[1] A. R. Aftabizadeh and N. H. Pavel, Boundary value problems for second order differential equations and a convex problem of Bolza, Differential Integral Equations 2 (1989), no. 4, 495509.

[2] __ Nonlinear boundary value problems for some ordinary and partial differential equations associated with monotone operators, J. Math. Anal. Appl. 156 (1991), no. 2, 535-557.

[3] S. Aizicovici and N. H. Pavel, Anti-periodic solutions to a class of nonlinear differential equations in Hilbert space, J. Funct. Anal. 99 (1991), no. 2, 387-408.

[4] N. C. Apreutesei, A boundary value problem for second order differential equations in Hilbert spaces, Nonlinear Anal. 24 (1995), no. 8, 1235-1246.

[5] Second-order differential equations on half-line associated with monotone operators, J. Math. Anal. Appl. 223 (1998), no. 2, 472-493.

[6] Continuous dependence on data for a second order evolution equation, Comm. Appl. Nonlinear Anal. 9 (2002), no. 1, 75-84.

[7] H. Attouch, Mésurabilité et monotonie, preprint, 1976, Univ. Paris XI, no. 183-76-53.

[8] - Variational Convergence for Functions and Operators, Applicable Mathematics Series, Pitman, Massachusetts, 1984.

[9] J.-P. Aubin, Approximation of Elliptic Boundary-Value Problems, Wiley-Interscience, New York, 1972.

[10] V. Barbu, A class of boundary problems for second order abstract differential equations, J. Fac. Sci. Univ. Tokyo Sect. IA Math. 19 (1972), 295-319.

[11] Sur un problème aux limites pour une classe d'équations différentielles non linéaires abstraits du deuxième ordre en t, C. R. Acad. Sci. Paris Sér. A-B 274 (1972), A459-A462 (French).

[12]_Existence theorems for a class of two point boundary problems, J. Differential Equations 17 (1975), 236-257.

[13] _ Nonlinear Semigroups and Differential Equations in Banach Spaces, Noordhoff International Publishing, The Netherlands, 1976.

[14] H. Brézis, Équations d'évolution du second ordre associées à des opérateurs monotones, Israel J. Math. 12 (1972), 51-60 (French).

[15] H. Brézis and A. Pazy, Convergence and approximation of semigroups of nonlinear operators in Banach spaces, J. Functional Analysis 9 (1972), 63-74.

[16] R. E. Bruck, Periodic forcing of solutions of a boundary value problem for a second-order differential equation in Hilbert space, J. Math. Anal. Appl. 76 (1980), no. 1, 159-173.

[17] R. Cristescu, Analiza Functionala, Editura Didactica si Pedagogica, Bucuresti, 1983.

[18] E. Mitidieri, Asymptotic behaviour of some second order evolution equations, Nonlinear Anal. 6 (1982), no. 11, 1245-1252.

[19] - Some remarks on the asymptotic behaviour of the solutions of second order evolution equations, J. Math. Anal. Appl. 107 (1985), no. 1, 211-221.

[20] G. Moroşanu, Second-order difference equations of monotone type, Numer. Funct. Anal. Optim. 1 (1979), no. 4, 441-450.

[21] E. I. Poffald and S. Reich, A quasi-autonomous second-order differential inclusion, Trends in the Theory and Practice of Nonlinear Analysis (Arlington, Tex, 1984), North-Holland Math. Stud., vol. 110, North-Holland, Amsterdam, 1985, pp. 387-392.

[22] _ An incomplete Cauchy problem, J. Math. Anal. Appl. 113 (1986), no. 2, 514-543.

[23] S. Reich and I. Shafrir, An existence theorem for a difference inclusion in general Banach spaces, J. Math. Anal. Appl. 160 (1991), no. 2, 406-412.

[24] L. Véron, Problèmes d'évolution du second ordre associés à des opérateurs monotones, C. R. Acad. Sci. Paris Sér. A 278 (1974), 1099-1101 (French). 


\section{Continuous dependence on data}

[25]_É_Euations non linéaires avec conditions aux limites de type Sturm-Liouville, An. Ştiinţ. Univ. Al. I. Cuza Iaşi Secț. I a Mat. 24 (1978), no. 2, 277-287 (French).

[26] Un exemple concernant le comportement asymptotique de la solution bornée de l'équation $d^{2} u / d t^{2} \in \partial \varphi(u)$, Monatsh. Math. 89 (1980), no. 1, 57-67 (French).

N. Apreutesei: Department of Mathematics, “Gh. Asachi” Technical University of Iasi, 700506 Iasi, Romania

E-mail address: napreut@math.tuiasi.ro 


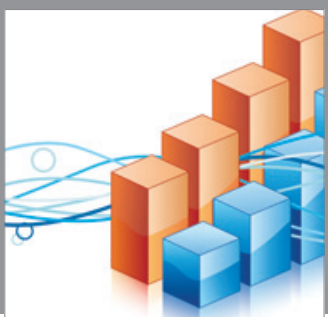

Advances in

Operations Research

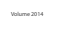

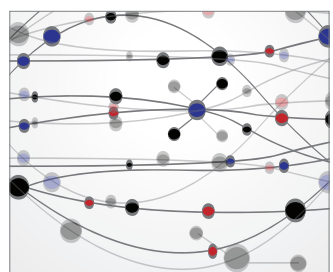

\section{The Scientific} World Journal
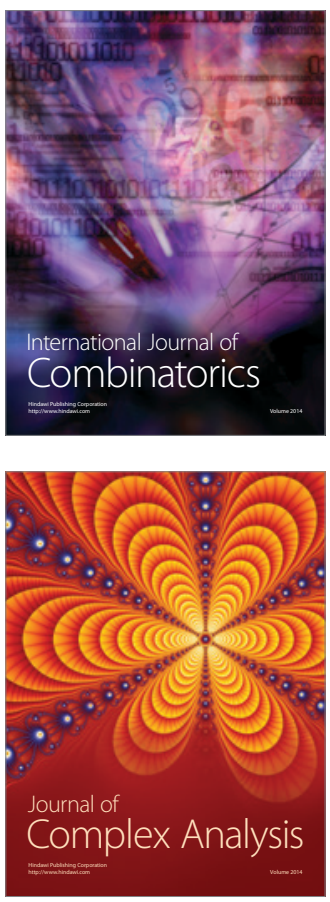

International Journal of

Mathematics and

Mathematical

Sciences
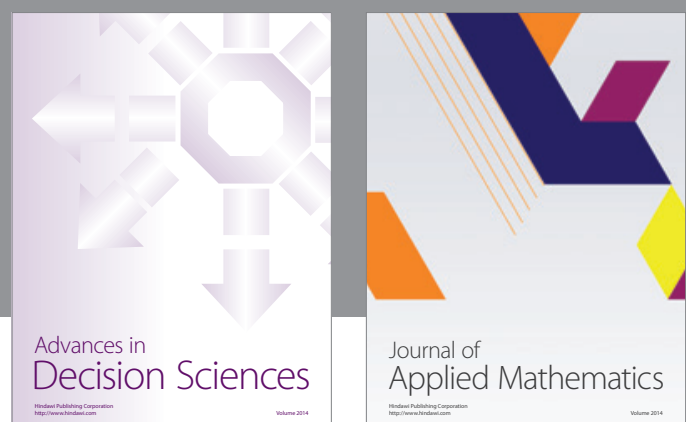

Journal of

Applied Mathematics
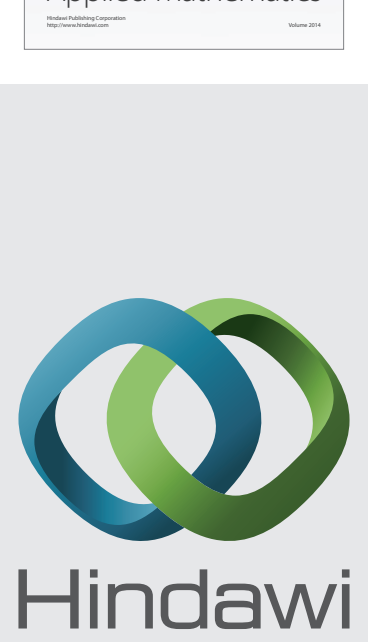

Submit your manuscripts at http://www.hindawi.com
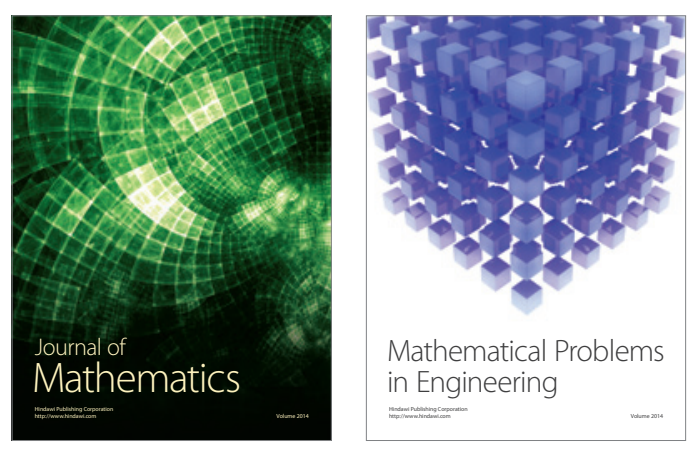

Mathematical Problems in Engineering
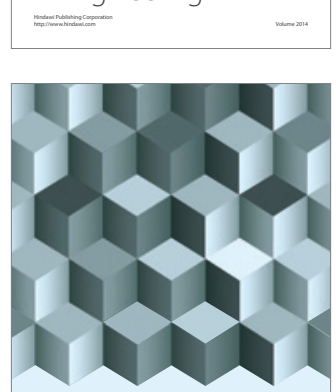

Journal of

Function Spaces
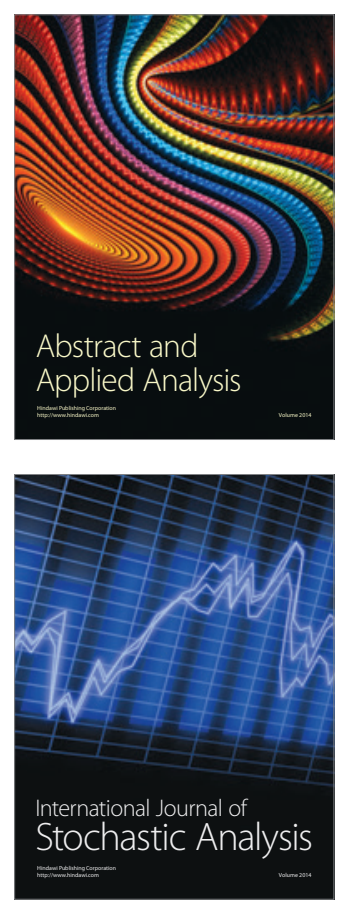

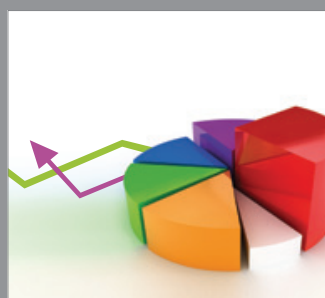

ournal of

Probability and Statistics

Promensencen
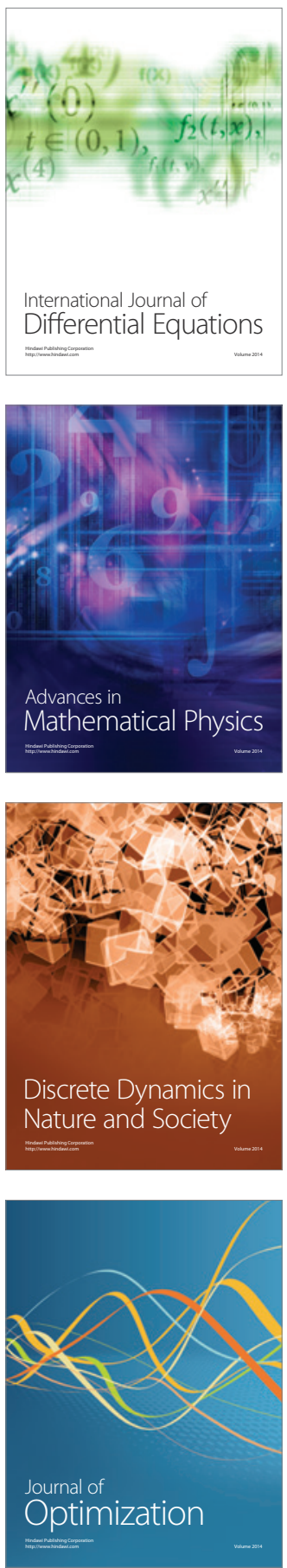